ARTÍCULO DE INVESTIGACIÓN

\title{
Estructura y productividad de los manglares en la reserva de biosfera Ría Celestún, Yucatán, México
}

\author{
Arturo Zaldívar Jiménez ${ }^{1}$ \\ Jorge Herrera Silveira ${ }^{1}$ \\ Carlos Coronado Molina ${ }^{2}$ \\ David Alonzo Parra ${ }^{3}$
}

\begin{abstract}
RESUMEN
Una zona importante de distribución de manglares en Yucatán está asociada al sistema lagunar de la Reserva de la Biosfera Ría Celestún, localizada al noroeste de la Península. Los principales impactos hacia este ecosistema son la interrupción de flujos de agua, el crecimiento urbano no controlado, el cambio en el uso del suelo y la deforestación. El suelo de la región es cárstico y altamente permeable; al no existir ríos, la única fuente de agua son la precipitación pluvial $(760 \mathrm{~mm}$ año-1) y las descargas de agua subterránea las cuales tienen su mayor influencia en la parte interna de la laguna, reflejándose en un gradiente de menor a mayor salinidad hacia la boca de la laguna que la conecta con el Golfo de México. Debido a este gradiente se observaron diferentes grados de estructura, dominancia de especies y productividad, registrándose las mayores estructuras y valores de caída de hojarasca de 4,09 $\mathrm{g} \mathrm{m}^{2}$ día en la parte interna y de 2,59 $\mathrm{g} \mathrm{m}^{2}$ día-1 en la zona de la boca. Temporalmente la productividad de la laguna está relacionada con la variabilidad climática de la región (nortes, secas y lluvias). Los diferentes patrones de estructura y producción de hojarasca fueron determinados por características del suelo como la salinidad y del clima. Por lo tanto las estrategias de manejo deben considerar estos aspectos, así como la variabilidad espacial y temporal de la hidrología, ya que modificaciones, incluso pequeñas, de este aspecto producirían impactos negativos de mayor escala, sugiriendo la vulnerabilidad de este ecosistema a los cambios hidrológicos y ambientales climáticos.
\end{abstract}

PALABRAS CLAVE:

Estructura, manglares, productividad, Ría Celestún, salinidad del suelo, Yucatán.

\begin{abstract}
An important zone of mangrove distribution in Yucatan is the Natural Protected Area Ría Celestún, located to the northwest of the Peninsula of Yucatan. The main impacts in this ecosystem are the interruption of water flows, uncontrolled urban growth, change in land use and deforestation. The soil of the region is karstic and highly permeable; as there are no rivers, the only source of water are the rainfall $\left(760 \mathrm{~mm}\right.$ year $\left.{ }^{-1}\right)$ and groudwater discharges as springs, which have its greater influence in the inner zone of the lagoon, being reflected in the gradient of lower seawater salinity in the inner zone and higher seawater salinity in the mouth that connects with the Gulf of Mexico. Due to this gradient, different mangrove forest structure patterns, dominant species and productivity were observed, recording the higher forest structure and productivity values de $4,09 \mathrm{~g} \mathrm{~m}^{2}$ day-1 in the inner zone and of $2,59 \mathrm{~m}^{2}$ day $^{-1}$ in the sea zone. The mangrove productivity in each zone of the lagoon is determined by climatic variability of the region ("nortes", dry and rain seasons). The different structure patterns and litter production were determined by soil salinity and climatic condition. Therefore, the management strategies should consider the structure and natural behavior and the spatial and tempo-ral variability of the mangrove ecosystems, and their vulnerability in the hydrology and climate change.
\end{abstract}

KEY WORDS:

Forest structure, mangrove forests, productivity, Ría Celestún, soil salinity, Yucatán. 


\section{INTRODUCCIÓN}

Los bosques de manglar dominan aproximadamente un $75 \%$ de la línea de costa en todo el mundo, se localizan entre los $30^{\circ} \mathrm{N}$ y los $30^{\circ} \mathrm{S}$ (Kangas y Lugo, 1990), son considerados sistemas altamente productivos, lo cual es muy variable entre los diferentes tipos de manglar, como resultado de los gradientes topográficos y las variaciones de la hidrodinámica (Pool et al., 1975; Twilley et al., 1986). Son diversos los factores que afectan la estructura y productividad de los bosque de manglar, entre los que se incluyen a la hidrología, la dinámica de nutrientes, el tipo de sedimentos y la salinidad del suelo (Lugo et al., 1988). Las características de la estructura, la productividad y la exportación de detritus a lo largo de un gradiente de hidrología y nutrientes definen cada uno de los tipos de manglar, de tal manera que la biomasa, la altura, la producción de hojarasca y la descomposición se incrementan del manglar chaparro al riverino o ribereño (Twilley y Day, 1999). En regiones costeras con clima seco y con déficit en la precipitación, en donde la amplitud de marea es baja (< $0,30 \mathrm{~m}$ ), la presencia de escurrimientos e infiltraciones de agua dulce del continente es la principal fuente de materiales incluyendo nutrientes, desempeñando un papel importante en el desarrollo de la comunidad de manglar (Smith, 1992; Twilley, 1998). La mayor parte de los estudios para evaluar la productividad de los manglares están basados en escalas de tiempo corto; en su mayoría, durante un ciclo anual.Aunque representan de manera general la productividad del sistema, no es posible identificar el comportamiento como resultado de las variaciones interanuales de la precipitación, escurrimientos de agua dulce, temperatura, salinidad del suelo y eventos climáticos de gran escala como tormentas y huracanes. En laguna de
Celestún, al estar localizada en un sistema cárstico y semiárido, se esperaría que las condiciones de la vegetación terrestre circundante (estructura y función) correspondan a manglares de zonas áridas con un pobre desarrollo de la estructura y producción de hojarasca, debido a la salinidad (condiciones semiáridas) y baja concentración de nutrientes inorgánicos (condiciones cársticas) principalmente fósforo en el suelo (Cintrón et al., 1978; Twilley, 1998). Es importante mencionar que el presente trabajo representa el primer programa de registro y evaluación de largo plazo en los manglares del Estado de Yucatán.

\section{OBJETIVOS}

Determinar la estructura y productividad de los manglares asociados a laguna Celestún a lo largo de un gradiente ambiental, así como la variación temporal que corresponde a épocas climáticas de la región, usando para ello datos de hojarasca colectados mensualmente, con lo cual se pretende clarificar los factores que determinan los patrones espaciales y temporales de producción de hojarasca en sistemas cársticos y semiáridos como los que presenta laguna Celestún.

\section{ÁREA DE ESTUDIO}

La laguna costera de Celestún, se localiza en el extremo noroeste de la Península de Yucatán, la parte norte de la laguna pertenece al Municipio de Celestún, en el Estado de Yucatán y la parte sur al municipio de Calkiní, en el Estado de Campeche. Se localiza entre los paralelos $20^{\circ} 45^{\prime}$ y $20^{\circ}$ 58' latitud norte y los meridianos $90^{\circ} 1^{\prime}$ y $90^{\circ}$ longitud oeste (Fig. 1). El clima de la región, es de tipo cálido semiárido con lluvias en verano y escasas todo el año 


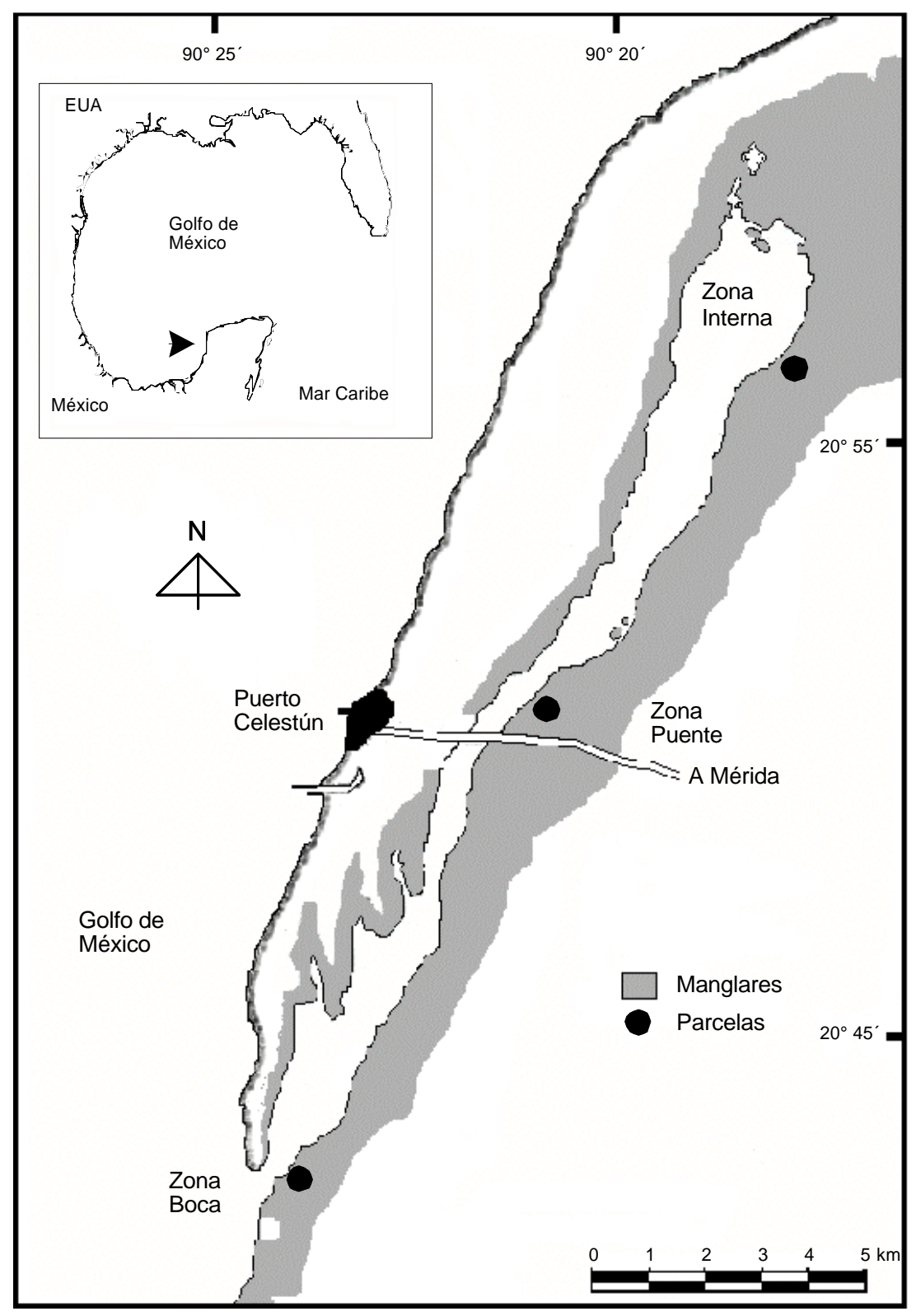

Figura 1. Localización de laguna Celestún y los sitios propuestos para los muestreos. 
(García y Mosiño, 1992). La temperatura promedio anual es de $28,5{ }^{\circ} \mathrm{C}$ siendo las

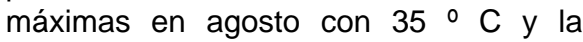
mínimas en febrero con $21^{\circ} \mathrm{C}$. La precipitación media anual es de $760 \mathrm{~mm}$. Los vientos dominantes son del sureste $(0 \mathrm{~km}$ $\left.\mathrm{h}^{-1}-15 \mathrm{~km} \mathrm{~h}^{-1}\right)$. Entre noviembre y febrero se presentan vientos intensos entre 50 $\mathrm{km} \mathrm{h}^{-1}$ - $90 \mathrm{~km} \mathrm{~h}^{-1}$, condición que es llamada localmente "nortes", ya que están asociados a frentes polares. El clima de la región está asociado a la variación de la precipitación, reconociéndose tres épocas climáticas: secas (marzo - mayo), lluvias (junio - octubre) y nortes (noviembre - febrero).

El suelo de la zona es de tipo calizo - cárstico de gran permeabilidad, lo que no permite escurrimientos a nivel de superficie; el agua filtrada aparece nuevamente en superficie por afloramientos del manto freático a manera de manantiales, que se localizan en los bordes y dentro de la laguna. La precipitación y los manantiales son las únicas fuentes de agua dulce con las que cuenta el sistema lagunar (Perry et al., 1995). En la figura 1 se presenta la localización de laguna Celestún y los sitios propuestos para los muestreos.

En laguna Celestún se reconocen tres zonas de acuerdo al gradiente de salinidad: la parte interna se caracteriza por los menores valores de salinidad en la columna de agua lagunar $\left(5 \mathrm{~g} \mathrm{~kg}^{-1}\right.$ a $12 \mathrm{~g}$ $\mathrm{kg}^{-1}$ ), esto se debe por la mayor influencia de las descargas subterráneas; en la parte sur se localiza la zona de la boca, caracterizada por salinidad marina (>30 g $\mathrm{kg}^{-1}$ ); la tercera zona localizada en el centro de laguna corresponde a la del puente, presenta valores intermedios de salinidad $\left(15 \mathrm{~g} \mathrm{~kg}^{-1}\right.$ a $\left.30 \mathrm{~g} \mathrm{~kg}^{-1}\right)$, es consi- derada una zona de mezcla entre zonas adyacentes (Herrera-Silveira, 1994).

\section{METODOLOGÍA}

Del manglar que crece en la margen continental de la laguna de Celestún y tomando en cuenta estudios previos (Herrera-Silveira et al., 1998), se eligieron tres sitios que corresponden a la zonificación de la laguna propuesta por HerreraSilveira (1994), considerando como criterio el comportamiento de la salinidad en la columna de agua. Los sitios de manglar seleccionados fueron la zona interna, puente y boca de la laguna (Fig. 1).

En cada zona se establecieron cuatro parcelas permanentes, dos localizadas en el borde de la laguna (manglar de franja) y dos hacia tierra adentro (manglar de cuenca); la composición florística, altura y diámetro a la altura del pecho (DAP) de todos los árboles mayores a 2,5 cm en DAP fueron registrados, las dimensiones de las parcelas fueron variables: para la zona interna y el puente, el área muestreada de cada parcela fue de 0,06 ha y para la zona de boca fue de 0,02 ha, debido a que la franja en esta zona es muy angosta $(<15$ $m$ ). En este trabajo se presentan los valores medios por zona. Las características estructurales: valores medios de DAP, densidad, área basal y altura fueron calculados siguiendo las recomendaciones de Cintrón y Shaeffer-Novelli (1984).

Para la medición de la caída de hojarasca en cada parcela se instalaron cinco canastas de $0,25 \mathrm{~m}^{2}$, teniendo un total de 20 canastas por cada zona. Las canastas fueron repartidas cubriendo el área de cada parcela. Las canastas se constru- 
yeron con tela de mosquitero de $1 \mathrm{~mm}$ de malla y fueron atadas por las cuatro esquinas a una altura promedio de 1,30 m sobre el piso del manglar, la colecta se llevó a cabo mensualmente. El material colectado fue secado $75^{\circ} \mathrm{C}$ en un tiempo promedio de 72 horas; posteriormente la muestra se separó y se pesó en dos partes: los componentes hojas y no hojas (estructuras reproductivas, ramas y misceláneo); la producción de hojarasca fue expresada en $\mathrm{g} \mathrm{m}^{-2}$ día $^{-1}$ (Brown, 1984).

En cada parcela se tomaron muestras de agua intersticial a $30 \mathrm{~cm}$ de profundidad para la medición de la salinidad, las muestras fueron tomadas con una jeringa dentro de tubos de pvc de 1 $\mathrm{m}$; los tubos fueron perforados en sus primeros $15 \mathrm{~cm}$ para permitir el libre flujo del agua intersticial. El agua intersticial fue colectada mensualmente, al mismo tiempo que la hojarasca. La salinidad del suelo fue medida con un refractómetro VISTA A366ATC y expresada en $\mathrm{g} \mathrm{kg}^{-1}$.

Para identificar diferencias espaciales y temporales en la producción de hojarasca, se aplicaron pruebas de ANDEVAS $(p<0,05)$ con diseño de Splitplot (Day et al., 1996). Se llevo a cabo un análisis a posteriori utilizando pruebas de contrastes lineales entre los diferentes tratamientos. Los análisis se llevaron a cabo con el paquete estadístico JMPâ versión 4.02 (SAS Institute, 2000). Adicionalmente, se obtuvieron registros de temperatura ambiente y precipitación a través de la Gerencia Regional de la Comisión Nacional del Agua para determinar qué factores ambientales influyen sobre la producción de hojarasca.

\section{RESULTADOS}

El desarrollo de la vegetación que bordea la laguna Celestún presentó un gradiente que corresponde a la zonifica- ción de la laguna: el mejor desarrollo de la vegetación de manglar se registró en la zona interna, mientras que hacia la boca de la laguna la estructura de la vegetación fue menor (Tabla 1). La zona interna, en cuanto a sus características estructurales difiere significativamente a las otras, ya que el diámetro $(F=73, p<0,05)$, área basal $(F=13, p<0,05)$, densidad $(F=$ $151, p<0,05)$ y altura $(F=434, p<0,05)$ fueron mayores.

Rhizophora mangle, Avicennia germinans y Laguncularia racemosa fueron las esepcies de mangle encontrados en los sitios de muestreo, Conocarpus erecta también fue observada aunque fuera de las parcelas, hacia áreas de tierra adentro. De manera general, la estructura de la comunidad del manglar que bordea la parte continental de laguna Celestún fue dominado por $R$. mangle con índice de valor de importancia (IVI) de $52 \%$, seguido por $A$. germinans con $26 \%$ y por ultimo con el $22 \%$ restante, L. racemosa. A nivel de zonas, en las áreas localizadas en el borde de la laguna (franja) se observaron cambios en la dominancia de las especies a lo largo de la laguna. En la zona interna, $L$. racemosa dominó la estructura de la comunidad con $61 \%$ de IVI, este valor disminuyó en dirección de la zona de la boca hasta alcanzar un valor medio de $5 \%$. Para el caso de $A$. germinans el comportamiento fue contrario, el aporte de esta especie a la comunidad en la zona interna fue el menor, con apenas el $6 \%$ del total, pero en la zona de la boca alcanzó su máximo desarrollo, dominando la estructura de la vegetación en un $75 \%$ (IVI). R. mangle, aunque de manera general dominó la estructura de la comunidad del manglar en laguna Celestún, registró su máximo desarrollo en la zona del puente dominando en un $51 \%$ el estrato arbóreo (Tabla 1).

Por otra parte, el patrón anual de producción de hojarasca presentó dos 
Tabla 1. Características estructurales de la vegetación de manglar en cada zona de laguna Celestún

\begin{tabular}{lccccc}
\hline ZONA & $\begin{array}{c}\text { DAP } \\
(\mathrm{cm})\end{array}$ & $\begin{array}{c}\text { ÁREA BASAL } \\
\left(\mathrm{m}^{2} \mathrm{ha}^{-1}\right)\end{array}$ & $\begin{array}{c}\text { DENSIDAD } \\
\left(\text { árbol ha }^{-1}\right)\end{array}$ & $\begin{array}{c}\text { ALTURA } \\
(\mathrm{m})\end{array}$ & No. SPP \\
\hline Interna & 17,4 & 41,5 & 1108 & 12,3 & 3 \\
\hline Puente & 10,6 & 23,6 & 1466 & 7,6 & 3 \\
\hline Boca & 10,2 & 26,0 & 3125 & 5,3 & 3 \\
\hline
\end{tabular}

periodos de máxima producción. El primero al final de la época de seca en el mes de mayo y otro en agosto y septiembre durante la lluvia (Fig. 2). Durante todos los meses, el mayor componente de la hojarasca fueron las hojas con aporte de entre $70 \%$ a $84 \%$ del total; el complemento de la hojarasca: tallos, misceláneo y estructuras reproductivas aportaron entre $16 \%$ y $30 \%$. En el período comprendido entre los meses de julio y diciembre se registro la mayor producción de estructuras reproductivas.

EI ANDEVA con Split-plot mostró diferencias significativas entre zonas $(F=$ $30, p<0,05)$, observándose un gradiente de productividad a lo largo de la laguna, el cual corresponde al mismo de la estructura de la vegetación; la zona más productiva fue la interna con media anual de 4,09 $\mathrm{g} \mathrm{m}^{2}$ día ${ }^{-1}$, seguido por la zona del puente con $3,16 \mathrm{~g} \mathrm{~m}^{2}$ día $\mathrm{a}^{-1}$ y 2,59 $\mathrm{g} \mathrm{m}^{2}$ día para la zona boca (Tabla 2).

En cuanto a la variabilidad temporal, se observaron diferencias significativas entre épocas $(F=174, p<0,05)$, la productividad en la temporada de nortes fue significativamente menor a las otras, registrándose valores en esta época de entre $2,09 \mathrm{~g} \mathrm{~m}^{2}$ día ${ }^{-1}$ y $2.52 \mathrm{~g} \mathrm{~m}^{2}$ día ${ }^{-1}$. Sin embargo, en la época de seca, se registró un incremento en la caída de hojarasca con valores medios entre $2,83 \mathrm{~g} \mathrm{~m}^{2}$ día $^{-1} \mathrm{y}$ $4,40 \mathrm{~g} \mathrm{~m}^{2}$ día $^{-1}$, pero fue hasta la temporada de lluvia donde se registró la mayor caída de hojarasca, con medias entre 2,85 $\mathrm{g} \mathrm{m}^{2}$ día ${ }^{-1}$ y 5,70 $\mathrm{g} \mathrm{m}^{2}$ día ${ }^{-1}$ (Tabla 2).

En cuanto al análisis de la salinidad del suelo, también se observó un gradiente de menor a mayor de la zona interna; la media anual de salinidad fue de $28,2 \mathrm{~g} \mathrm{~kg}^{-1}$ con valores mínimos y máximos de $9 \mathrm{~g} \mathrm{~kg}^{-1}$ y $43 \mathrm{~g} \mathrm{~kg}^{-1}$, respectivamente, mientras que para la zona del puente la media anual fue de $45,8 \mathrm{~g} \mathrm{~kg}^{-1}$ $\left(17 \mathrm{~g} \mathrm{~kg}^{-1}\right.$ y $\left.70 \mathrm{~g} \mathrm{~kg}^{-1}\right) \mathrm{g} \mathrm{kg}^{-1}$ y en la boca de $64 \mathrm{~g} \mathrm{~kg}^{-1}$ (34 $\mathrm{g} \mathrm{kg}^{-1}$ a $97 \mathrm{~g} \mathrm{~kg}^{-1}$ ), valores promedio, mínimo y máximo.

La producción de hojarasca esta fuertemente relacionada con la salinidad del suelo y la precipitación (Fig. 2). En mayo se observo un pico de producción de hojarasca el cual coincide con la mayor media mensual de salinidad del suelo (55 g kg-1), este mes correspondió a la época de seca.

En septiembre se registró un segundo pico de producción de hojarasca, el cual corresponde a la máxima precipitación mensual; durante este mes la adición de estructuras reproductivas (flores y frutos) fue mayor, con un aporte hasta de $30 \%$ de la producción total del mes. La temperatura está más asociada a la variación climática de la región, registrándose las temperaturas más altas durante la temporada seca y las más bajas durante los nortes. 

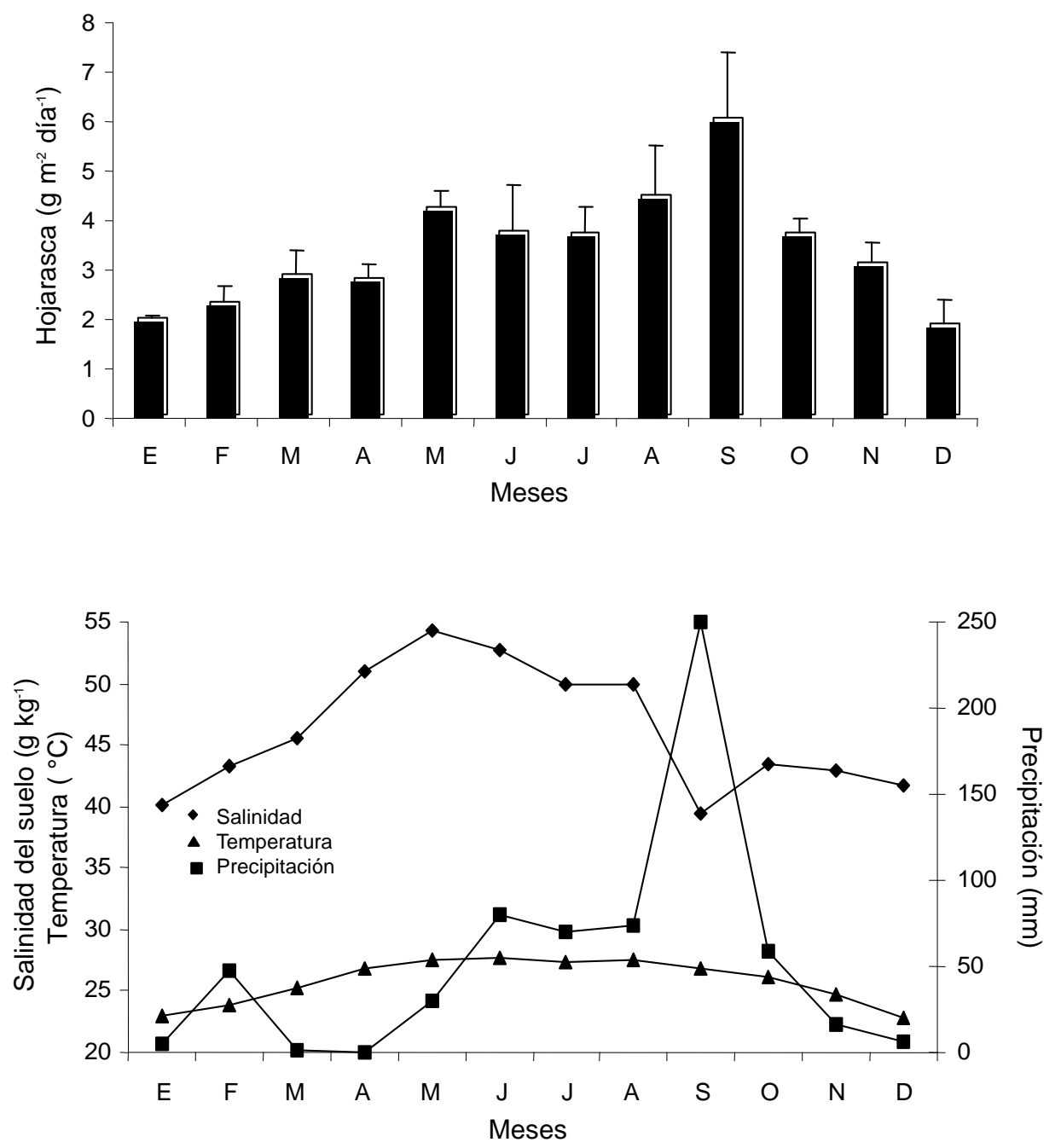

Figura 2. Patrón anual de producción de hojarasca y la relación entre la salinidad del suelo, precipitación total y temperatura promedio del aire. 
Tabla 2. Análisis de contrastes donde se muestran diferencias en la productividad $\left(\mathrm{g} \mathrm{m}^{-2}\right.$ día $\left.{ }^{-1}\right)$ entre zonas y épocas.

La media \pm error estándar con diferente letra muestra diferencias significativas $(p<0,05)$

\begin{tabular}{lccc}
\hline ZONA & INTERNA & PUENTE & BOCA \\
\hline Productividad & $4,09(0,52)^{\mathrm{a}}$ & $3,16(0,33)^{\mathrm{b}}$ & $2,59(0,24)^{\mathrm{c}}$ \\
\hline Zona - época & Nortes $^{\prime}$ & Seca & Lluvias $^{\mathrm{a}}$ \\
\hline Interna & $2,52(0,19)^{\mathrm{c}}$ & $4,40(0,47)^{\mathrm{b}}$ & $5,70(0,67)^{\mathrm{a}}$ \\
\hline Puente & $2,23(0,07)^{\mathrm{b}}$ & $2,89(0,03)^{\mathrm{b}}$ & $4,37(0,42)^{\mathrm{a}}$ \\
\hline Boca & $2,09(0,19)^{\mathrm{b}}$ & $2,83(0,11)^{\mathrm{a}}$ & $2,85(0,69)^{\mathrm{a}}$ \\
\hline
\end{tabular}

\section{DISCUSIÓN}

La estructura de la vegetación de manglar registrada en Celestún por su origen geológico y localización es clasificada como manglar de tipo cuenca y franja, pero por sus características funcionales como producción primaria puede ser clasificada en tres tipos, según la clasificación de Lugo y Snedaker (1974): el manglar de la zona interna por su alta productividad y estructura puede ser considerado como manglar de tipo riverino, la zona del puente como cuenca y como manglar de franja en la zona boca.

El gradiente de salinidad observado en Celestún es un factor asociado con la estructura de la vegetación y dominancia de las especies, ya que en la zona interna con mayor influencia de agua subterránea presenta bajas salinidades (media de $28,2 \mathrm{~g} \mathrm{~kg}^{-1}$ ) siendo la especie dominante $L$. racemosa, la que se caracteriza por ser mejor competidora en ambientes de baja salinidad y alto contenido de nutrientes (Mitsch y Gosselink, 1993), sugiriendo que la concentración de este ultimo es mayor comparado con el de otras zonas de la laguna.
La salinidad del suelo y las características del clima desempeñan un papel importante en la variabilidad temporal de la producción de hojarasca. Para la región donde la escasa precipitación y la temperatura alta favorecen los procesos de evaporación y un incremento en la salinidad del suelo, el manglar se encuentra sometido a estrés hídrico y salino (Medina, 1998). Esto también se ha observado en manglares asociados a ríos como el caso de Laguna de Términos, Campeche (Day et al., 1996). Durante la época de mayor precipitación los manglares producen la mayor cantidad de propágulos, ya que durante esta época se registran las mayores inundaciones favoreciendo la dispersión de los mismos.

Las características cársticas no permiten la presencia de ríos en Yucatán, por lo que debido a la escasa precipitación y al clima seco en laguna Celestún, la presencia de manantiales adquiere mayor importancia como fuente de nutrientes y flujo de agua que mantiene diluida la salinidad (Herrera-Silveira et al., 1998). La zona interna está fuertemente influenciada por descargas a través de 
manantiales y, conforme se avanza hacia la boca, la influencia se hace menor, esto explica el gradiente espacial de estructura y productividad (Zaldívar, 2004).

Aunque para describir los patrones de producción a largo plazo, solamente se utilizaron la salinidad, la temperatura y la precipitación, es necesario tomar en cuenta factores como velocidad del viento, radiación, evaporación y otras características del clima además de la concentración de nutrientes y los factores que producen estrés del suelo de tipo anhídrico sulfúrico, así como cuantificar espacial y temporalmente la influencia de las descargas subterráneas, para esclarecer los factores ambientales que determinan los patrones de producción de hojarasca en sistemas cársticos y semiáridos.

\section{CONCLUSIONES}

Los manglares a lo largo de laguna Celestún muestran diferentes patrones de estructura y producción de hojarasca, las características del suelo; lasalinidad y el clima de la región son los principales controladores.

Factores ambientales como salinidad del suelo, descargas de agua subterránea, lluvias, tormentas y la variabilidad de estos con respecto al patrón climático de la región, son de importancia en la estructura de la vegetación y en la producción de hojarasca en la laguna Celestún. Por lo tanto las estrategias de manejo deben considerar su estructura y su funcionamiento natural, a sí como la variabilidad espacial y temporal del ecosistema de manglar y su vulnerabilidad a cambios en la hidrología y clima.

La salinidad del suelo es un factor espacial que explica en gran parte los diferentes patrones de estructura y producción, pero, como siguiente paso se debe determinar la dinámica de nutrientes, inundación y estresores como anhídrido y sulfúrico que serían importantes para esclarecer cómo y cuáles son los factores ambientales que influyen sobre el desarrollo de los manglares en sistema cársticos.

Las evaluaciones y registros de los ecosistemas de manglar son también necesarios, en especial y para nuestro interés en los manglares asociados a laguna Celestún, ya que representan un tipo especial de manglar como resultado de la combinación de características ambientales locales y regionales. El buen estado de salud y la conservación de los manglares en la parte continental de la laguna Celestún, con base en su estructura y productividad, sugiere que la mejor estrategia de manejo es la conservación. La dinámica natural de los manglares en esta área debe ser considerada como sitio de referencia para estudios posteriores, de aquí la importancia de las áreas naturales protegidas.

\section{RECONOCIMIENTOS}

Al personal del Laboratorio de Producción Primaria del CINVESTAV-IPN Mérida, en especial a la Tec. Suemy Chi Chan y a la Biól. Claudia Teutli, por el soporte técnico en la elaboración del trabajo de campo. A DUMAC A.C. Yucatán por permitir el uso de sus instalaciones en la estación de campo en Celestún. Este proyecto fue financiado por el Programa de Monitoreo de Humedales Costeros a cargo del Laboratorio de Producción Primaria.

\section{REFERENCIAS}

Brown, M. 1984. Mangrove litter production and dynamics. In: S.C. Snedaker y J.G. Snedaker, (eds). The mangrove ecosystem: Research 
methods. Monographs on Oceanographic Methodology 8. UNESCO/SCOR. Reino Unido. $\mathrm{p}: 231-237$.

Cintrón, G. y Y. Shaeffer-Novelli. 1984. Methods for studying mangrove structure. In: S.C. Snedaker y J.G. Snedaker (eds). The mangrove ecosystem: Research methods. Monographs on Monographs on Oceanographic Methodology 8. UNESCO/ SCOR. Reino Unido. p:91-13.

Day, J.W.; C. Coronado-Molina; F.R. Vera-Herrera; R. Twilley; V.H. Rivera-Monroy; H. Alvarez-Guillen; R. Day y W. Conner. 1996. A 7-year record of above-ground net primary production in a southeastern Mexican mangrove forest. Aquatic Botany 27: 267-284.

García, E. y P. Mosiño. 1992. Los climas de México. Instituto de Geografía de la UNAM. México, D.F. 27 p.

Herrera-Silveira, J.A. 1994. Spatial heterogeneity and seasonal patterns in a tropical coastal lagoon. J. Coastal Res. 10(3):738-746.

Herrera-Silveira, J.A.; J. Ramírez y A. Zaldívar. 1998. Overview and characterization of the hydrology and primary producers communities of selected coastal lagoons of Yucatan, Mexico. Aquatic Ecosystem Health and Management 1:353-372.

SAS Institute Inc. 2000. Statistical Discovery Software, JMPâ v 4.02. SAS Institute Inc. Cary, N.C. 635 p.

Kangas, P.C. y E.A. Lugo. 1990. The distribution of mangrove and salt marsh in Florida. Tropical Ecology 31(1):32-39.
Lugo, A.E. y S.C. Snedaker. 1974. The ecology of mangrove. Annu. Rec. Ecol. Syst. 5:39-64.

Lugo, A.E.; S. Brown y M.M. Brinson. 1988. Forested wetlands in freshwater and salt-water environments. Limnology and Oceanography 33:894-909.

Medina, E. 1998. Mangrove physiology: the challenge of salt, heat and light stress under recurrent flooding. Anais do IV Simposio de Ecosistemas Brasileiros V:25-56.

Mitsch, W.J. y J.G. Gosselink. 1993. Wetlands. Van Nostrand Reinhold. Second Edition. Nueva York. 699 p.

Perry, E.; L. Marín y J. McClain. 1995. Ring of Cenotes (sinkholes), northwest Yucatan, Mexico: its hydrogeologic caracteristics and possible association with the Chicxulub impact crater. Geology 23(1):17-20.

Pool, D.J.; A.E. Lugo y S.C. Snedaker. 1975. Litter production in mangrove forests of southern Florida and Puerto Rico. In: G. Walsh; S. Snedaker y Teas (eds). Proceeding of the International Symposium on Biology and Management of Mangrove. $\mathrm{H}$. Institute of Food and Agriculture Science. University of Florida. Gainesville. p:213-237.

Smith, T.J. 1992. Forest Structure. In: A.I. Robertson y D. M. Alongi (eds). Tropical mangrove ecosystems. American Geophysical Union. Washington D.C. p:101-136.

Twilley, R.R. 1998. Mangrove wetlands. In: Messina, M.G. y W.H. Conner (eds). Southern Forested Wetlands. Ecology and Management. Lewis 
Publishers. Boca Raton, Florida. p:445-473.

Twilley, R.R.; A.L. Lugo y C. PattersonZucca. 1986. Litter production and turnover in basin mangrove forest in Southwest Florida. Ecology 67(3):670-683.

Twilley, R.R. y J.W. Day. 1999. The productivity and nutrient cycling of mangrove ecosystem. In: YanezArancibia, A. y A.L. Lara-Domínguez (eds). Ecosistemas de manglar en
América Tropical. Instituto de Ecología, A.C. México. UICN/ HORMA, Costa Rica, NOAA/NMFS Silver Spring MD. p:127-152.

Zaldívar, A. 2004. Cambios en los patrones de estructura y productividad en manglares sometidos a gradientes ambientales en una laguna costera cárstica en la Península de Yucatán (SE, México). Tesis de Maestría. CINVESTAV-IPN Unidad Mérida. Mérida, Yucatán, México. 78 p.» en la reserva de la biosfera Ría Celestún, Yucatán, México. Madera y Bosques Número especial 2:25-35. 